\title{
Neutrophil-lymphocyte ratio and mean platelet volume as predictive factors for liver fibrosis and steatosis in patients with chronic hepatitis B
}

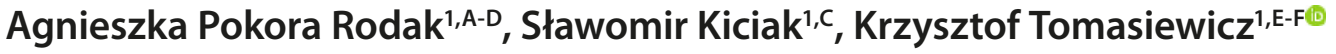 \\ 1 Department and Clinic of Infectious Diseases, Medical University of Lublin, Poland \\ A - Research concept and design, B - Collection and/or assembly of data, C - Data analysis and interpretation, \\ $D$ - Writing the article, E-Critical revision of the article, $F$ - Final approval of article
}

Pokora Rodak A, Kiciak S, Tomasiewicz K. Neutrophil-lymphocyte ratio and mean platelet volume as predictive factors for liver fibrosis and steatosis in patients with chronic hepatitis B. Ann Agric Environ Med. 2018; 25(4): 690-692. doi: 10.26444/aaem/99583

\begin{abstract}
Objective. Evaluation of the neutrophil-lymphocyte ratio and mean platelet volume as predictive factors for liver fibrosis and steatosis in HBV patients qualified for antiviral treatment.

Materials and method. The study comprised $38 \mathrm{CHB}$ patients who had commenced antiviral treatment, and 20 healthy volunteers who constituted the clinical control group. All patients had their blood count taken and underwent hepatic assessment using transient elastography with CAP (controlled attenuation parameter).

Results. It was found that the mean hepatic fibrosis was $8.7 \mathrm{kPa}( \pm 8.8)$ and the mean liver steatosis $-286 \mathrm{db} / \mathrm{m}( \pm 64)$. Mean NLR - 2.78( \pm 1.1$)$, whereas in the control group the mean NLR value was 1.64( \pm 0.98$)$. A negative linear correlation $(r=-0.34$; $p=0.035$ ) was found between liver fibrosis and the NLR value in the study group. No correlation was observed between hepatic steatosis and the NLR. Mean MPV - 12.6f ( \pm 3.1$)$, which was considerably higher in the CBH patients than in the control group. A positive correlation $(r=0.79, p=0.001)$ was found between MPV and disease severity evaluated with transient elastography.

Conclusions. The NLR and the MPV were significantly higher in the CHB patients than in the healthy volunteers. Both the NLR and the MPV can be treated as predictive factors for liver fibrosis in this group of patients.
\end{abstract}

Key words

neutrophil-lymphocyte ratio, hepatitis B, liver fibrosis, liver steatosis

\section{INTRODUCTION}

The HBV virus is a carcinogen. It induces hepatocellular carcinoma, which is among the top-five most-frequent cancers in the population. The virus leads to liver cirrhosis and failure. Researchers continue to seek specific markers used for disease progression assessment and the determination of the inflammation course (the so-called staging). Recently, a few studies have pointed to the importance of the NLR as a potential predictive factor for chronic hepatitis B progression and liver failure [1]. Attention was drawn to the possibility of predicting acute-on-chronic hepatitis B using the NLR in patients with the disease which were not necessarily caused by the virus [2]. There are reports which describe the use of the NLR as a predictive factor of mortality in patients with acute-on-chronic HBV [3]. The neutrophil-to-lymphocyte ratio (NLR) is used as a simple, cheap and readily accessible marker for the assessment of the condition of patients with chronic and oncological diseases. Recently, several reports have noted that the NLR can also prove useful to predict the disease progression in the case of chronic hepatitis B. In chronic hepatitis B, the NLR was evaluated in this study as a predictive factor for hepatic fibrosis and steatosis. Attempts were made to introduce the NLR into the diagnostics and monitoring of such diseases as Hodgkin's lymphoma [4, 5, 6], diffuse large B-cell Lymphoma [7], gastric cancer or acute

Address for correspondence: Agnieszka Pokora Rodak, Department and Clinic of Infectious Diseases, Medical University of Lublin, Poland

e-mail: agniecha811@gmail.com

Received: 18.07.2018; accepted: 05.11.2018; first published: 04.12.2018 venous thrombosis $[8,9,10,11]$. Furthermore, everyday clinical practice is faced with the so-far underestimated problem of liver steatosis. The literature still lacks consistent data concerning fatty liver in patients with HBV, yet most of the papers show that HBV patients display less advanced stages of steatosis than the non-infected population $[12,13$, 14]. Hence the subject of this study, the aim of which was to determine whether NLR values are significantly varied in HBV patients and healthy individuals. Furthermore, it attempts to show whether there is a correlation between the NLR and hepatic fibrosis or steatosis in HBV patients. MPV is a parameter which relates to the standard CBC test. Hence it is easily accessible, replicable, and cheap. The evaluation of MPV levels is usually overlooked in clinical practice. However, the source literature provides several examples which stress the importance of this parameter and suggest that it is correlated with the disease's severity $[15,16]$.

\section{OBJECTIVE}

Evaluation of the neutrophil-lymphocyte ratio as a predictor for liver fibrosis and steatosis in non-treated patients with chronic hepatitis B.

\section{MATERIALS AND METHOD}

This retrospective study was conducted in 2017 at the Department of Infectious Diseases of Independent Public 
Hospital No 1 of the Medical University in Lublin, eastern Poland. It involved patients with newly diagnosed chronic $\mathrm{HBV}$ in whom HBs antigen and anti-HBe and HBV DNA had been found. The study comprised patients with $\mathrm{CHB}$ who had commenced antiviral treatment. HAV, HCV and HIV infections were ruled out. The patients with cardiovascular, respiratory, endocrine and renal problems, or neoplastic processes, were excluded from the study. Patients with BMI greater than 25 were also excluded. None of the patients smoked cigarettes or abused alcohol, according to the criteria of the Dietary Guidelines for Americans for the years 20152020.

Study group characteristics: the average age of the patients was $48.2( \pm 12)$; women constituted $48 \%$ of the study group and men $52 \%$; the control group comprised 20 healthy individuals with an average age of $42.1( \pm)$; women constituted $40 \%$ of the control group and men $60 \%$.

Transient Elastography (TE) was conducted by two qualified researchers. Blood for $\mathrm{CBC}$ was collected on the same day. The level of fibrosis and steatosis, as well as the controlled attenuation parameter, were measured simultaneously using transient elastography with a medium-sized probe. Normal range for MPV - 8-11.9 fl.

MS Office 2007 suite and Statistica v. 10.0 software by StatSoft were used for statistical analysis of the gathered results. Moreover, mean, median and maximum and minimum values were provided for the statistical description of the results. Normal distribution compliance was checked with the Shapiro-Wilk test.

Comparative analysis for the examined variables was conducted using the non-parametric U Mann-Whitney statistical test for two trials. The correlations between particular variables were described with the use of the Spearman correlation test. The identified differences were considered statistically significant at the significance level of $\mathrm{p}<0.05$.

\section{RESULTS}

The study showed a statistically significant negative correlation between fibrosis and NLR in HBV patients who had not yet received antiviral treatment. Additionally, a considerable difference in the NLR value was determined in the control group, in which it amounted to 1.2. In the study group, the NLR value was 1.6. The mean steatosis level was $255 \mathrm{~dB} / \mathrm{m}$ and remained at the S2 level (17). No relationship was found between the steatosis (CAP) and the NLR level (Tab.1; Fig. 1). The mean MPV was $12.6 \mathrm{fl}( \pm 3.1)$, and was considerably higher in the $\mathrm{CBH}$ patients than in the control group. A positive correlation $(\mathrm{r}=0.79 ; \mathrm{p}=0.001)$ was found between MPV and disease severity evaluated with transient elastography (Tab. 2; Fig. 2). No relationship was identified between MPV levels and liver steatosis.

Table 1. Statistical values of CAP, NLR, Fibrosis

\begin{tabular}{lcccc}
\hline Whole group & N-valid & R-Spearman & $t(N-2)$ & $p$ \\
\hline NLR \& CAP & 38 & 0.145947 & 0.88516 & 0.38 \\
\hline NLR \& FIBROSIS & 38 & -0.342807 & -2.18952 & 0.035 \\
\hline
\end{tabular}


of death [3]. The patients included in the presented study were not at risk of liver failure, which was confirmed by a Fibroscan examination. Similar to the work by H. Liu et al, the current study found a negative correlation between NLR and disease aggravation [3]. J. Gong et al. presents results consistent with the presented observations, indicating that NLR is reversely correlated with disease aggravation [11]. Higher values of this marker were observed in more severely sick patients. Morover, this indicator can be used as predictor of mortality from liver failure in the course of chronic severe hepatitis B. The current study demonstrates that there is no correlation between NLR and liver steatosis. In 2015, H. Yilmaz et al. published a study which indicated that NLR could act as a very good predictive factor of fibrosis in non-alcoholic steatohepatitis [20]. The source literature lacks study results which would show a correlation between NLR and the level of steatosis. N. Moreau et al quotes researchers arguing that NLR value is not linked to disease severity in HBV patients, and emphasises that NLR should be treated as an independent non-invasive predictive marker [2]. Moreover, MPV values were significantly higher in the $\mathrm{CHB}$ patients than in the individuals in the control group. A positive correlation was found between MPV and disease severity evaluated with transient elastography. This result is similar with the results of E. Karagoz and P. Ye $[15,16]$.

\section{CONCLUSIONS}

The NLR value is higher in HBV patients than in healthy individuals. It can be used as a predictive factor of disease severity in patients with chronic HBV. NLR has more value in combination with transient elastography assessment.

\author{
Abbreviations: \\ CHB - chronic hepatitis B \\ CAP - controlled attenuation parameter \\ NLR - neutrophil-lymphocyte ratio \\ MPV - mean platelet value \\ TE - Transient Elastography \\ $\mathrm{HBV}$ - hepatitis B virus \\ CBC - cell blood count \\ $\mathrm{kPa}$ - kilopascal \\ Fibr - Fibrosis \\ NAFLD - non- alcoholic fatty liver disease \\ $\mathrm{Fl}$ - femtolitr
}

\section{REFERENCES}

1. Fan Y, Li X, Zhou XF, et al. Value of neutrophil-lymphocyte ratio in predicting hepatitis B-related liver failure. Zhonghua Gan Zang Bing Za Zhi 2017; 25: 726-731.

2. Moreau N, Wittebole X, Fleury Y, et al. Neutrophil-to-Lymphocyte Ratio Predicts Death in Acute-on-Chronic Liver Failure Patients
Admitted to the Intensive Care Unit: A Retrospective Cohort Study. Shock. 2018; 49: 385-392.

3. Liu H, Zhang H, Wan G, et al. Neutrophil-lymphocyte ratio: a novel predictor for short-term prognosis in acute-on-chronic hepatitis B liver failure. J Viral Hepat. 2014; 21: 499-507.

4. Romano A, Parrinello NL, Vetro C, et al. Prognostic meaning of neutrophil to lymphocyte ratio (NLR) and lymphocyte to monocyte ration (LMR) in newly diagnosed Hodgkin lymphoma patients treated upfront with a PET-2 based strategy. Ann Hematol. 2018; 97(6): 10091018.

5. Marcheselli R, Bari A, Tadmor T, et al. Neutrophil-lymphocyte ratio at diagnosis is an independent prognostic factor in patients with nodular sclerosis Hodgkin lymphoma: results of a large multicenter study involving 990 patients. Hematol Oncol. 2017; 35: 561-566.

6. Reddy J, Hernandez M, Gunther J, et al. Pre-treatment neutrophil/ lymphocyte ratio and platelet/lymphocyte ratio are prognostic of progression in early stage classical Hodgkin lymphoma. Haematol. 2018; 180: 545-549.

7. Go SI, Park S, Kim JH, et al. A New Prognostic Model using the NCCN-IPI and Neutrophil-to-Lymphocyte Ratio in Diffuse Large B-Cell Lymphoma. Tumori. 2017: tj5000694. doi: 10.5301/tj.5000694.

8. Yao C, Zhang Z, Yao Y, et al. Predictive value of neutrophil to lymphocyte ratio and platelet to lymphocyte ratio for acute deep vein thrombosis after total joint arthroplasty: a retrospective study. Orthop Surg Res. 2018; 13: 40.

9. Kim H, Ro SM, Yang JH, et al. The neutrophil-to-lymphocyte ratio prechemotherapy and postchemotherapy as a prognostic marker in metastatic gastric cancer. Korean J Intern Med. 2018. doi: 10.3904/ kjim.2016.293.

10. Ming L, Jiang Z, Ma J. Platelet-to-lymphocyte ratio, neutrophil-tolymphocyte ratio, and platelet indices in patients with acute deep vein thrombosis. 2018; 47: 143-147.

11. Gong J, Liang YL, Zhou W, et al. Prognostic value of neutrophil-tolymphocyte ratio associated with prognosis in $\mathrm{HBV}$-infected patients. J Med Virol. 2018; 90: 730-735.

12. Zhong GC, Wu YL, Hao FB, et al. Current but not past hepatitis B virus infection is associated with a decreased risk of nonalcoholic fatty liver disease in the Chinese population: A case-control study with propensity score analysis. J Viral Hepat. 2018. doi: 10.1111/jvh.12878.

13. Xiong J, Zhang H, Wang Y, et al. Hepatitis B virus infection and the risk of nonalcoholic fatty liver disease: a meta-analysis. Oncotarget. 2017; 8: 107295-107302.

14. Joo EJ, Chang Y Yeom JS, et al. Hepatitis B virus infection and decreased risk of nonalcoholic fatty liver disease: A cohort study. Hepatology. 2017; 6: 828-835.

15. Karagoz E, Ulcay A, Tanoglu A, et al. Clinical usefulness of mean platelet volume and red blood cell distribution width to platelet ratio for predicting the severity of hepatic fibrosis in chronic hepatitis B virus patients. Eur J Gastroenterol Hepatol. 2014; 26: 1320-4.

16. Pan Y, Muheremu A, Wu X. Relationship between platelet parameters and hepatic pathology in patients with chronic hepatitis B infection - a retrospective cohort study of 677 patients. J Int Med Res. 2016; 44: 779-86.

17. Shen F, Zheng R, Mi YQ, et al. Controlled attenuation parameter for non-invasive assessment of hepatic steatosis in Chinese patients. World J Gastroenterol. 2014; 20: 4702-11.

18. Yilmaz B, Aydin H, Can G, et al. The relationship between fibrosis level and blood neutrophil to lymphocyte ratio in inactive hepatitis $\mathrm{B}$ carriers. Eur J Gastroenterol Hepatol. 2014; 26: 1325-8.

19. Peng Y, Li Y, He Y, et al. The role of neutrophil to lymphocyte ratio for the assessment of liver fibrosis and cirrhosis: a systematic review. Expert Rev Gastroenterol Hepatol. 2018; 12: 503-513.

20. Yilmaz H, Yalcin KS, Namuslu M, et al. Neutrophil-Lymphocyte Ratio (NLR) Could Be Better Predictor than C-reactive Protein (CRP) for Liver Fibrosis in Non-alcoholic Steatohepatitis(NASH). Ann Clin Lab Sci. 2015 ; 45(3): 278-86. 